\title{
Fast Processing of Optical Fringe Movement in Displacement Sensors Without Using an ADC
}

\author{
Babar HUSSAIN ${ }^{1,3 *}$, Taj MUHAMMAD ${ }^{1}$, Muhammad REHAN ${ }^{2}$, Haroon AMAN ${ }^{1}$, \\ Muhammad ASLAM ${ }^{1}$, Masroor IKRAM ${ }^{2}$, and M. Yasin Akhtar RAJA ${ }^{3}$ \\ ${ }^{1}$ National Institute of Lasers and Optronics, P. O. 45650, Islamabad, Pakistan \\ ${ }^{2}$ Pakistan Institute of Engineering and Applied Sciences, P. O. 45650, Islamabad, Pakistan \\ ${ }^{3}$ Department of ECE, EPIC Building, UNC Charlotte, 9201 University City Blvd. Charlotte, NC 28223-000 \\ *Corresponding author: Babar HUSSAIN_Email: bhussai1@uncc.edu
}

\begin{abstract}
An interferometer based optical sensor for displacement measurement is reported. This method requires quite simple signal processing as well as least electronic components. Referring to this technique, two photodiodes spatially shifted by 90 degrees were used. The output of photodiodes was converted into rectangular signals which were extracted in LabVIEW using the data acquisition card without using an analog to digital converters (ADC). We have also processed the signals in $\mathrm{C}++$ after acquiring via parallel port. A Michelson interferometer configuration was used to produce linear fringes for the detection of displacements. The displacement less than $100 \mathrm{~nm}$ could be measured using this technique.
\end{abstract}

Keywords: Fast signal processing, optical displacement sensor, direction detection, Michelson interferometer

Citation: Babar HUSSAIN, Taj MUHAMMAD, Muhammad REHAN, Haroon AMAN, Muhammad ASLAM, Masroor IKRAM, et al., "Fast Processing of Optical Fringe Movement in Displacement Sensors Without Using an ADC," Photonic Sensors, DOI: 10.1007/s13320-013-0088-5.

\section{Introduction}

Displacements or vibrations of mechanical parts of a machine or large buildings range from a few micrometers to a few centimeters. Sometimes, such movements are precisely monitored to avoid any mechanical damage. Conventional vibration sensors (mechanical/electrical) are used to detect vibration amplitudes within a few millimeter ranges. If higher accuracy is required, optical sensors take place of conventional sensors. Several optical methods, for example, classical interferometry [1-3], holographic interferometry [3], speckle interferometry [4] and shadow moiré [5] have been demonstrated and reported in the literature.

Laser Doppler vibrometers (LDVs) are considered as very accurate sensors for the measurement of motion over a wide range of frequencies and amplitudes [6]. The accuracy of 他 the LDV is based on interferometry in which two coherent light beams interfere to produce fringes. One is a reference beam, and the other is the subject or signal beam. These two beams form linear optical fringes, when overlapped, due to interference effects. The signal beam is partially reflected from the object under observation. The movement of the surface of the object under test is translated into the fringe displacement, and the optical signal can be

Received: 28 July 2012 / Revised version: 26 January 2013

(C) The Author(s) 2013. This article is published with open access at Springerlink.com 
converted into the electronic signal using one or more photo-detectors. The signals from photodetectors are processed digitally after converting them from the analog to digital format using an analog to digital converter (ADC). There are a number of factors involved in the accuracy and speed of the measurement [7-10]. The overall performance of an LDV is affected by a variety of potential error sources arising from optical, electronic and digital processing sections of the system [11].

Classical interferometry is mostly used in vibration measurement systems because it can be used for the detection of the real time displacement and vibration of a surface [12-14]. The vibration of the amplitude less than $1 \mathrm{~nm}$ and displacement of the order of several meters can be measured depending on the coherence length of the laser being used. The signal from the optical setup can be sent over a long distance using fiber optic cables of course with challenges of its own [15].

Recently, we reported an analog processing based vibration measurement technique [16]. In this paper, we demonstrate a vibration detection technique in which signal processing can be performed without using an $\mathrm{ADC}$, therefore, real time information about a vibrating surface can be obtained at a much faster rate as compared to other techniques. A significant amount of the processing time can be saved because an ADC could take up to several milliseconds in analog to digital conversion of one cycle.

\section{Experimental setup}

The schematic of the setup used for the experiments is shown in Fig. 1. Light from the coherent source is divided into two coherent beams using a beamsplitter (BS). One of the beams (reference beam) is reflected from the mirror $\mathrm{M}_{1}$. This mirror is slightly tilted to form spatially linear fringes. $M_{2}$ is the reflecting and vibrating surface that moves in the direction along $\mathrm{a}-\mathrm{b}$. A mirror can also be mounted on the vibrating surface for this purpose. These two beams reflected from $\mathrm{M}_{1}$ and $\mathrm{M}_{2}$ are overlapped after passing through the beamsplitter again and form interference fringes shown in Fig. 2. A diverging lens or magnification optics can be used to enlarge the fringe pattern. The larger size of the fringe pattern helps to detect the signals using two photodiodes $\left(\mathrm{PD}_{1}\right.$ and $\left.\mathrm{PD}_{2}\right)$ placed in spatial quadrature.
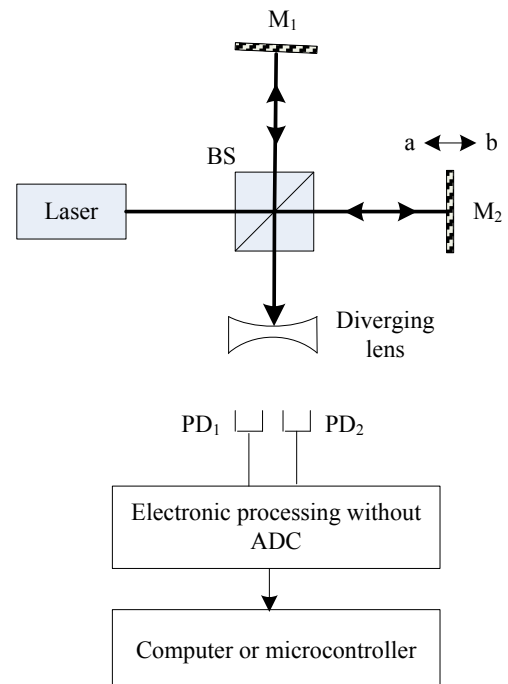

Fig. 1 Block diagram of the displacement sensor.

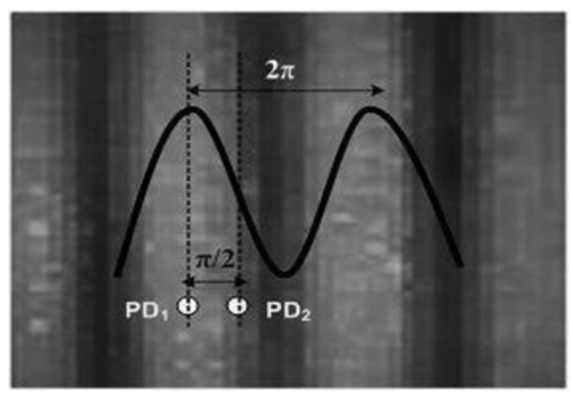

Fig. 2 Fringe pattern

\section{Description}

The optical path difference (OPD) is the key factor which is exploited in interferometer based sensors to measure the displacement, pressure, temperature etc $[17,18]$. Figure 2 shows that the two photodiodes are fixed at a phase separation of $\pi / 2$ with respect to the intensity profile of the fringe pattern. Let us assume that $L_{1}$ and $L_{2}$ are the OPDs corresponding to $\mathrm{PD}_{1}$ and $\mathrm{PD}_{2}$, respectively. The 
phase of the interference signal detected by $\mathrm{PD}_{1}$ can be given as

$$
\alpha(t)=\frac{2 \pi}{\lambda} L_{1}(t)
$$

where the constant phase difference between the outputs of the two photodiodes is

$$
\varphi=\frac{2 \pi}{\lambda}\left(L_{2}-L_{1}\right)
$$

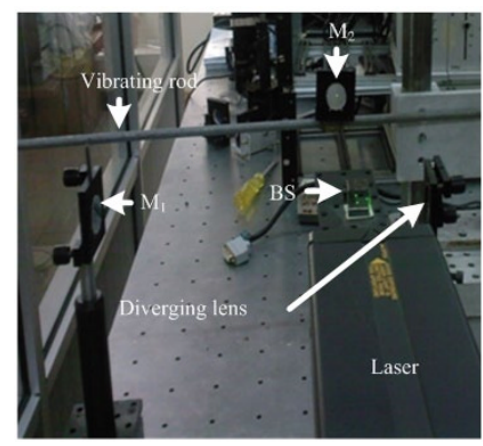

Fig. 3 Optical setup for vibration detection.

In our technique (the setup is shown in Fig. 3), the two photodiodes are adjusted precisely using a micro-mechanical arrangement such that

$$
\varphi \cong \pm\left(\frac{\pi}{2}+n \pi\right), n=0,1,2, \cdots
$$

If the fringe spacing $(F S)$ is too small, higher values of $n$ can be selected to adjust the photodiodes. However, as the fringe spacing is reduced, a smaller area photodiode is required. The fringe spacing is given by

$$
P S=\frac{\lambda}{2 \sin \frac{\theta}{2}} \times F
$$

where $\lambda$ is the wavelength of light, $\theta$ is the angle between two interfering beams, and $F$ is the factor by which the fringe pattern spreads out depending on the focal length of the lens or a lens system and the distance of photodiodes from the lens. The angle between the two interfering beams can be controlled by the tilt of the mirror $\mathrm{M}_{1}$. When the fringes move due to the vibration, the relative phase between the two output signals will be either $+\pi / 2$ or $-\pi / 2$ depending on the placement of the photodiodes.

The electronics for detection of the displacement consists of an amplifier and a comparator to convert the sinusoidal signals from photodiodes to rectangular waves. The same circuit is used for both diodes. The two diodes are spatially shifted by 90 degrees with respect to each other. The simulation is performed in Proteus software using two sine waves instead of photodiodes. The result shows that two square waves corresponding to the two photodiodes are out of phase by $90^{\circ}$ as shown in Fig. 4.

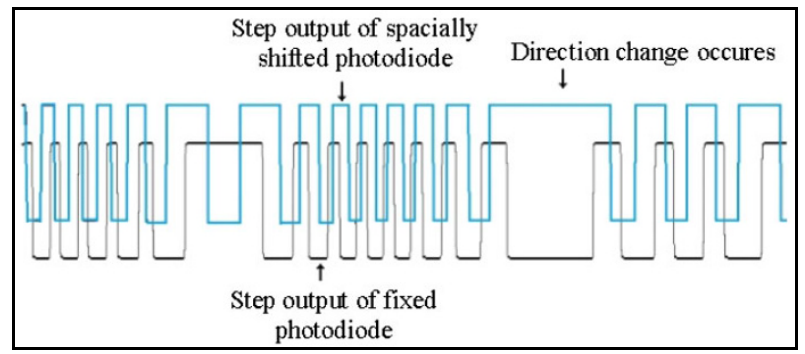

Fig. 4 Output of the circuit for detection of the direction of the displacement.

\section{Analyzing displacement signal}

We used LabVIEW and $\mathrm{C}++$ for processing the vibration signal from the interferometer using the data acquisition card and PC parallel port, respectively. The front panel of the LabVIEW VI is illustrated in Fig. 5. Logical methodology used for extracting the current direction of the vibrating surface is shown in the flowchart in Fig. 6. The Mathscript tool in LabVIEW was used to implement the flow chart. Table 1 explains possible outputs of the comparators regarding two photodiodes for two different directions $a$ and $b$ during the vibration of the surface.

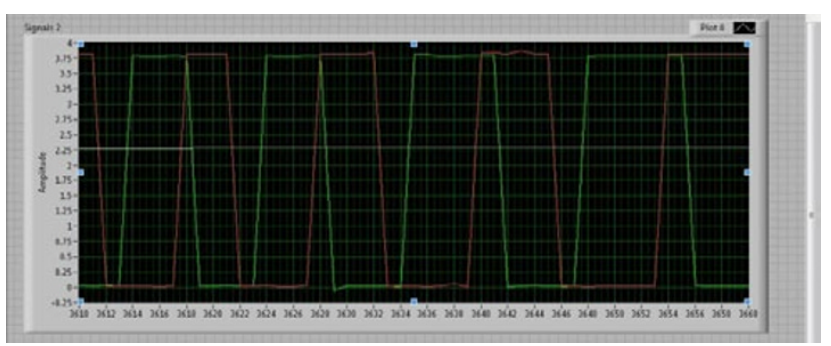

Fig. 5 Front panel of LabVIEW VI for extracting the displacement signal.

Step outputs of the comparators shown in Fig. 4 can be processed in the computer or microcontroller 
to interpret the direction of movement during vibration. Therefore, the amplitude as well as frequency of the vibration can be found with the high accuracy and speed without using an ADC.

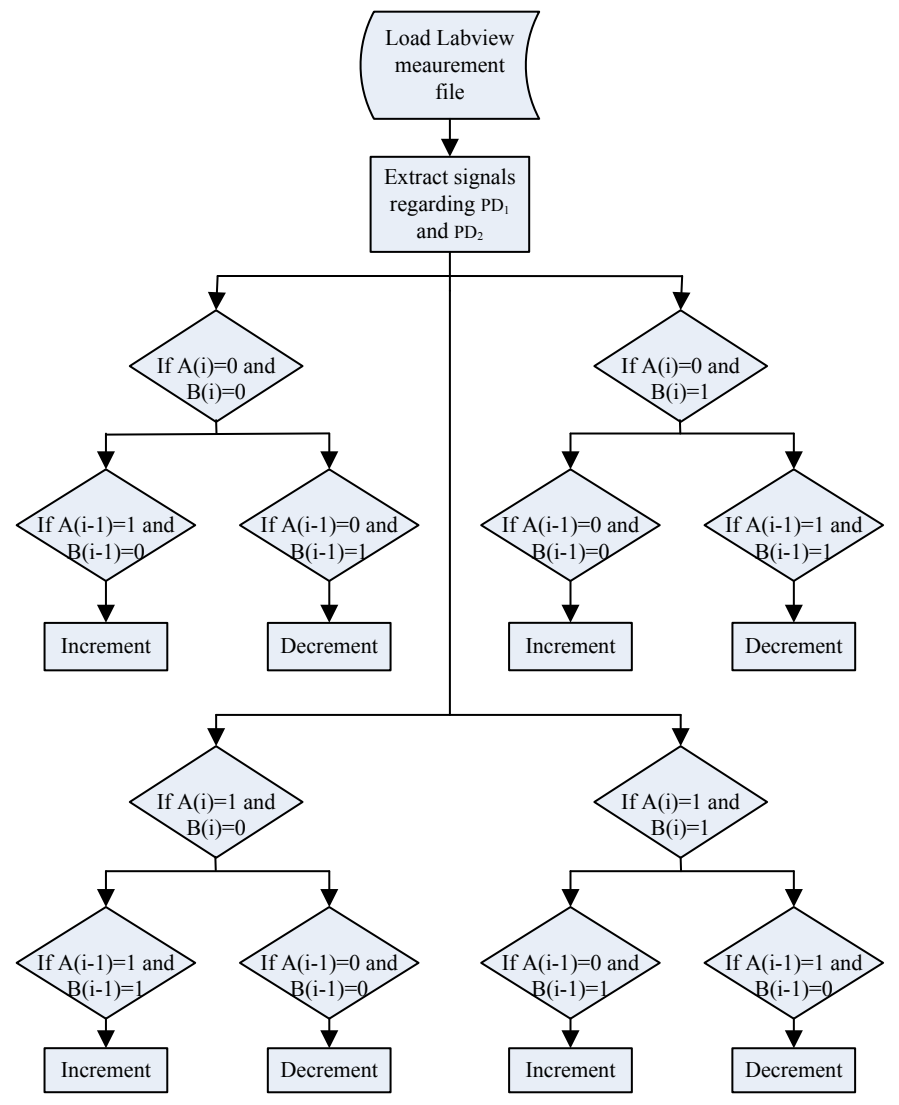

Fig. 6 Flow chart of the code for displacement measurement.

Table 1 Sequential step outputs of two photodiodes.

\begin{tabular}{c|c|c|c}
\hline \multicolumn{2}{c|}{ For direction a } & \multicolumn{2}{c}{ For direction b } \\
\hline $\mathrm{A}\left(\mathrm{PD}_{1}\right)$ & $\mathrm{B}\left(\mathrm{PD}_{2}\right)$ & $\mathrm{A}\left(\mathrm{PD}_{1}\right)$ & $\mathrm{B}\left(\mathrm{PD}_{2}\right)$ \\
\hline 1 & 0 & 0 & 0 \\
\hline 1 & 1 & 0 & 1 \\
\hline 0 & 1 & 1 & 1 \\
\hline 0 & 0 & 1 & 0 \\
\hline
\end{tabular}

\section{Conclusions}

In this technique of fringe detection, we have used two photodiodes spatially shifted by 90 degrees phase separation. The output of the photodiodes is converted into rectangular signals which are extracted in LabVIEW using the data acquisition card. We have also processed the signals in $\mathrm{C}++$ after acquiring the signals via parallel port. The technique is very simple, and signal conditioning and data acquisition can be performed in much faster rates as compared to other techniques. Therefore, it is very suitable to apply in real-time feedback of the vibrating objects and surfaces. Further work is in progress to improve the accuracy of the techniques and also miniaturize the system for portability and ruggedness.

Open Access This article is distributed under the terms of the Creative Commons Attribution License which permits any use, distribution, and reproduction in any medium, provided the original author(s) and source are credited.

\section{References}

[1] Y. N. Ning, K. T. V. Grattan, A. W. Palmer, and B. T. 
Meggit, "A novel detection scheme for vibration monitoring using optical techniques," Optics and Lasers in Engineering, vol. 15, no. 2, pp. 115-126, 1991.

[2] J. R. Baker, R. I. Laming, T. H. Wilinshurt, and N. A. Halliwell, "A new high sensitivity laser vibrometer," Optics and Laser technology, vol. 22, no. 4, pp. 241-244, 1990.

[3] K. Wier, W. J. O. Boyle, B. T. Meggit, A. W. Palmer, and K. T. V. Grattan, "A novel adaptation of the Michelson interferometer for the measurement of vibration," Journal of Lightwave Technology, vol. 10, no. 5, pp. 700-703, 1992.

[4] C. Shakher, A. L. Vyas, and A. Seth, "Real time monitoring of vibrations using interferometric sensor," in Proceedings of International Conference on Communications and Signal, vol. 2, pp. 1185-1188, 1997.

[5] S. Prakash, I. P. Singh, and C. Shakher, "Display of tilt information of vibrating object in time average mode using lateral shearing interferometry and interferometric grating," Optics and Laser Technology, vol. 33, no. 2, pp. 117-120, 2001.

[6] J. J. Dosch and D. M. Lally, "A heterodyne laser interferometer for primary calibration of accelerometers," in Proceedings of 21st IMAC Conference on Structural Dynamics, Kissimmee, Florida, pp. 977-984, 2003.

[7] O. D. Rochefoucauld, S. M. Kannah, and E. S. Olson, "Recording depth and signal competition in heterodyne interferometry," Journal of the Acoustical Society of America, vol. 117, no. 3, pp. 1267-1284, 2005.

[8] L. Yarovoi and G. Siegmund, "The effect of three-wave interference in laser Doppler vibrometry," Measurement Science and Technology, vol. 15, no. 10, pp. 2150-2156, 2004.
[9] C. Rembe and A. Draebenstedt, "Laser-scanning confocal vibrometer microscope: theory and experiments," Review of Scientific Instruments, vol. 77, no. 8, pp. 083702-1-083702-11, 2006.

[10] M. Bauer, F. Ritter, and G. Siegmund, "High-precision laser vibrometers based on digital Doppler signal processing," in Proc. SPIE, vol. 4827, pp. 50-61, 2002.

[11] G. Siegm und, "Sources of measurement error in laser Doppler vibrometers and proposals for unified specifications," in Proc. SPIE, vol. 7098, pp. Y1-Y13, 2008.

[12] L. B. Yuan, "Recent progress of in-fiber integrated interferometers," Photonic Sensors, vol. 1, no. 1, pp. $1-5,2011$.

[13] Y. J. Rao, "Study on fiber-optic low-coherence interferometric and fiber Bragg grating sensors," Photonic Sensors, vol. 1, no. 4, pp. 382-400, 2011.

[14] Y. Jiang and W. H. Ding, "Recent developments in fiber optic spectral white-light interferometry," Photonic Sensors, vol. 1, no. 1, pp. 62-71, 2011.

[15] T. K. Gangopadhyay, "Non-contact vibration measurement based on an extrinsic Fabry-Perot interferometer implemented using arrays of single mode fibres," Measurement Science and Technology, vol. 15, no. 5, pp. 911-917, 2004.

[16] B. Hussain, M. Ahmed, G. Hussain, M. Saleem, and M. Nawaz, "Analog processing based vibration measurement technique using Michelson interferometer," Photonic Sensors, DOI: 10.1007/s13320-012-0061-8, 2012.

[17] L. B. Yuan and Y. T. Dong, "Loop topology based white light interferometric fiber optic sensor network for application of perimeter security," Photonic Sensors, vol. 1, no. 3, pp. 260-267, 2011.

[18] Q. X. Yu and X. L. Zhou, "Pressure sensor based on the fiber optic extrinsic Fabry-Perot interferometer," Photonic Sensors, vol. 1, no. 1, pp. 72-83, 2011. 